12

\title{
Применение формулы Донкина в теории отражающих и поворотных устройств
}

\author{
() Ю.К. Голиков, ${ }^{1}$ А.С. Бердников, ${ }^{2, \uparrow}$ А.С. Антонов, ${ }^{2,3}$ Н.К. Краснова, ${ }^{1}$ К.В. Соловьёв ${ }^{1,2}$ \\ ${ }^{1}$ Санкт-Петербургский политехнический университет Петра Великого, \\ 195251 Санкт-Петербург, Россия \\ ${ }^{2}$ Институт аналитического приборостроения РАН, \\ 190103 Санкт-Петербург, Россия \\ ${ }^{3}$ Физико-технический институт им. А.Ф. Иоффре РАН, \\ 194021 Санкт-Петербург, Россия \\ ฯ e-mail: asberd@yandex.ru
}

Поступило в Редакцию 20 мая 2018 г.

В окончательной редакции 27 мая 2018 г.

Принято к публикации 28 мая 2018 г.

Электростатическими поворотными устройствами называются электронно- и ионно-оптические элементы, изменяющие направление движения параллельного монохроматического пучка заряженных частиц на желаемый угол с сохранением параллельности пучка. Принцип подобия траекторий для электрических полей, однородных по Эйлеру, обеспечивает выполнение этого свойства при использовании полей с нулевым порядком однородности. Формула Донкина для трехмерных однородных гармонических функций обеспечивает доступ к максимально широкому классу аналитических выражений, задающих однородные электрические потенциалы нулевого порядка. Рассмотрен алгоритм синтеза электростатических поворотных систем, преобразующих входные параллельные монохроматические пучки в выходные параллельные монохроматические пучки. Алгоритм основан на формуле Донкина и обеспечивает гарантированную устойчивость пучка при малых отклонениях от плоскости симметрии электрического поля.

Ключевые слова: электрические поля, функции, однородные по Эйлеру, принцип подобия траекторий в оптике заряженных частиц, формула Донкина, аналитические решения уравнения Лапласа, отражающие и поворотные системы.

DOI: 10.21883/JTF.2019.12.48496.201-18

\section{Введение}

Настоящая работа продолжает исследования электронно-оптических свойств электрических полей, однородных по Эйлеру с нулевым порядком однородности, которые были начаты в публикациях $[1,2]$. Задачей, которая рассматривается в настоящей работе, является конструирование поворотных и отражающих систем с помощью электрических полей, однородных по Эйлеру с нулевой степенью однородности и обеспечивающих устойчивость пучка при его малых смещениях от плоскости симметрии устройства.

Электрические поля, однородные по Эйлеру со степенью однородности, равной $n$, обязаны удовлетворять тождеству

$$
\mathbf{E}(\lambda x, \lambda y, \lambda z) \equiv \lambda^{n-1} \mathbf{E}(x, y, z)
$$

где $\lambda>1$ - произвольный множитель. Такие электрические поля являются вектор-функциями, положительно однородными по Эйлеру в том узком смысле, который придается этому термину в математическом анализе $[3,4]$. Электрические поля нулевой степени однородности характеризуются электрическими потенциалами вида

$$
U(x, y, z)=V(x, y, z)+U_{0} \ln \left(z+\sqrt{x^{2}+y^{2}+z^{2}}\right),
$$

где $V(x, y, z)$ - однородная скалярная функция нулевой степени, удовлетворяющая тождеству $V(\lambda x, \lambda y, \lambda z) \equiv$ $\equiv V(x, y, z)$, а $U_{0}-$ произвольная константа $[5,6]$. Чтобы функция (2) удовлетворяла уравнению Лапласа, необходимо и достаточно, чтобы уравнению Лапласа удовлетворяла функция $V(x, y, z)$.

Вид фиксированной логарифмической добавки, использованной в (2), можно варьировать в зависимости от рассматриваемой задачи, хотя в математическом смысле все такие выражения будут эквивалентны друг другу $[5,6]$. Однако любая логарифмическая поправка привносит сингулярность электрического потенциала в начале координат. Это существенным образом искажает форму электродов в окрестности этой точки и в конечном итоге делает систему электродов неконструктивной. Поэтому будем считать, что рассматриваемые здесь электрические потенциалы вида (2) являются сами по себе однородными функциями нулевой степени, и что для них логарифмическая добавка (2) равна нулю. (Не исключено, впрочем, что какие-то интересные в практическом смысле полевые конфигурации, описываемые 
формулами (2), могут быть упущены из рассмотрения при таком подходе.)

Потенциал электрического поля должен удовлетворять трехмерному уравнению Лапласа, и этот факт совместно с требованием однородности по Эйлеру делает нахождение выражений общего вида для подобных потенциалов нетривиальной задачей. В частности, единственным планарным потенциалом с нулевой степенью однородности является $U_{0}(x, y)=U_{0} \operatorname{arctg} y / x$, a единственным осесимметричным потенциалом с нулевой степенью однородности является $U(z, r)=$ $=U_{0} \ln \left(r /\left(z+\sqrt{r^{2}+z^{2}}\right)\right)$ [7]. Поэтому перспективы разнообразия электронно-оптических и ионно-оптических систем, использующих однородные электрические поля нулевой степени, выглядят, вообще говоря, не слишком обнадеживающими.

Тем не менее переход от вырожденных двумерных полей к полноценным трехмерным полям обеспечивает достаточно широкие возможности для варьирования однородных электрических полей нулевой степени и делает синтез и оптимизацию электронно-оптических и ионно-оптических систем, использующих подобные электрические поля, осмысленной задачей. Любые трехмерные гармонические функции, однородные по Эйлеру с нулевой степенью однородности, могут быть представлены с помощью формулы Донкина как

$$
V(x, y, z)=F\left(\frac{x}{z+\sqrt{x^{2}+y^{2}+z^{2}}}, \frac{y}{z+\sqrt{x^{2}+y^{2}+z^{2}}}\right),
$$

где $F(p, q)$ - произвольная функция, удовлетворяющая двумерному уравнению Лапласа $\partial^{2} F / \partial p^{2}+\partial^{2} F / \partial q^{2}=$ $=0[1,2,8-13]$. А класс двумерных гармонических функций $F(p, q)$ достаточно разнообразен, чтобы сделать исследование подобных электронно- и ионно-оптических систем интересной задачей.

Легко проверить, что при подстановке в формулу (3) произвольной гармонической функции $F(p, q)$ получается гармоническая однородная функция $V(x, y, z)$ нулевой степени. Обратное утверждение также справедливо: для любой гармонической однородной функции $V(x, y, z)$ нулевой степени найдется подходящая двумерная гармоническая функция $F(p, q)$, с помощью которой функция $V(x, y, z)$ может быть представлена в виде (3). При этом разным двумерным гармоническим функциям $F(p, q)$ соответствуют разные однородные гармонические функции $V(x, y, z)$ нулевой степени, и эта связь взаимно-однозначна.

При практическом использовании формулы (3) можно применять весь аппарат аналитических функций комплексного переменного $f(z)=f(x+i y)=F(x, y)+$ $+i G(x, y)$, тесно связанных с решениями двумерного уравнения Лапласа. Вещественные функции $F(x, y)$ и $G(x, y)$ будут гармоническими, а для любой двумерной гармонической функции $F(x, y)$ либо $G(x, y)$ существует прототип в виде аналитической функции комплексного переменного $f(z)$, вещественной либо мнимой частью которого является заданная гармоническая функция. Для того чтобы для потенциала вида (3) плоскость $y=0$ была плоскостью симметрии (т. е. чтобы траектория частицы, запущенной в плоскости $O X Z$, удовлетворяющей условию $y=0$, и дальше оставалась в той же плоскости), необходимо и достаточно, чтобы гармоническая функция $F(p, q)$ была симметричной по переменной $q$ : $F(p,-q)=F(p,+q)$. Техника манипулирования аналитическими функциями комплексного переменного $f(z)$ и синтез с их помощью электрических полей с нужными характеристиками рассматривается в [14]. Одним из полезных применений этой техники является синтез ахроматических транспортирующих систем со скрещенными электростатическими полями, рассмотренный в [15].

В настоящей работе рассматривается применение электрических полей, однородных по Эйлеру с нулевой степенью однородности, для создания поворотных систем (электростатических зеркал), которые преобразуют входные параллельные монохроматические пучки заряженных частиц в выходные параллельные монохроматические пучки заряженных частиц. Специфика таких систем состоит в том, что в них поворот пучка осуществляется на угол, приближающийся к $180^{\circ}$, а конфигурация электродов, которая осуществляет поворот пучка, должна быть компактна и занимать небольшую область пространства. Некоторые системы такого рода уже рассматривались в публикациях $[1,2]$. Задачей же настоящей работы является синтез таких электродных конфигураций, оперирующих параллельными пучками в плоскости симметрии устройства, которые обеспечивают гарантированную устойчивость параллельных пучков при малых отклонениях от плоскости симметрии в вертикальном направлении.

\section{1. Постановка задачи}

Для движения заряженных частиц в электрических полях, являющихся однородными по Эйлеру со степенью однородности $n$, справедлив принцип подобия траекторий $[16,17]$. А именно если масштабировать начальные координаты заряженной частицы в $\lambda$ раз, начальную кинетическую энергию масштабировать в $\lambda^{n}$ раз и сохранить углы вылета заряженной частицы неизменными, то каким бы ни был коэффициент масштабирования $\lambda>0$, новая траектория заряженной частицы будет представлять собой исходную траекторию, геометрически масштабированную в $\lambda$ раз как единое целое. В частности, в силу принципа подобия траекторий, электрические поля с ненулевыми степенями однородности могут использоваться как инструмент для создания энергоспектрографов с идеальными спектрографическими свойствами $[18,19]$.

При нулевой степени однородности электрического поля принцип подобия траекторий принимает специфический вид. В этом случае масштабированию подверга- 
ются только начальные координаты, а начальная кинетическая энергия остается в неизменном виде. Рассмотрим пробную одиночную траекторию с начальной точкой, лежащей в дрейфовом пространстве и не совпадающей с началом координат. Подвергнем ее начальные условия операции масштабирования (в смысле принципа подобия), меняя коэффициент масштабирования $\lambda$ в некотором непрерывном интервале значений. Результатом будет пучок заряженных частиц, которые стартуют с некоторым разбросом точек старта по координате под одним и тем же начальным углом и с одинаковыми начальными кинетическими энергиями. Принцип подобия гарантирует, что все полученные траектории будут результатом геометрического масштабирования исходной траектории. Поэтому если конечная точка у исходной траектории также лежит в дрейфовом пространстве, так, что траектория на этом участке представляет собой прямую линию, то и остальные траектории монохроматического пучка на выходе системы будут представлять собой прямые линии, параллельные друг другу.

Следовательно, электростатическое поле с потенциалом, являющимся однородной по Эйлеру функцией с нулевым показателем однородности, будет преобразовывать входной параллельный моноэнергетический пучок в выходной параллельный моноэнергетический пучок, отклоняя его на некоторый угол. Угол поворота пучка является функцией от энергии пучка. Подобные системы можно использовать в качестве монохроматоров, выделяющих из параллельного пучка частиц с разбросом по энергии пучок, характеризуемый заданной энергией, а также в качестве энергоанализаторов, если снабдить устройство дополнительной широкоугольной линзой, фокусирующей параллельный пучок в точку детектирования. В равной степени электронно-оптические и ионно-оптические системы с однородными по Эйлеру электрическими полями нулевой степени могут использоваться в качестве эффективно работающих электростатических призм [20].

Отдельной областью практического применения данного эффекта является использование электростатических зеркал с однородными по Эйлеру электрическими полями нулевой степени в качестве поворотных и отражающих систем $[1,2]$. А именно поворотными устройствами будем считать электронно- и ионно-оптические элементы, целенаправленно изменяющие направление движения параллельного монохроматического пучка заряженных частиц на желаемый угол с сохранением параллельности пучка. Дополнительным условием, как правило, является большая величина угла поворота, иногда приближающаяся к $180^{\circ}$. Также для поворотных систем характерно требование малых геометрических размеров области пространства, в которой осуществляется поворот пучка.

В практике синтеза электронно-оптических и ионнооптических систем необходимость в поворотных системах как специальных элементах конструкции возникает довольно редко. Но в именно таких случаях хорошие оптические характеристики и, что самое главное, геометрическая компактность устройства являются серьезным вызовом для разработчика, особенно принимая во внимание, что общая теория поворотных систем, вообще говоря, не слишком популярна. В качестве примера можно привести квазиконический энергоанализатор $[18,21-$ 23], в котором в силу специфики конструкции применение компактной и эффективно работающей поворотной системы является жизненной необходимостью: здесь инжекция анализируемых электронов внутрь диспергирующего электростатического поля должна осуществляться изнутри длинного и узкого осесимметричного канала, ограниченного электродами устройства, причем под большим углом поворота пучка по отношению к оси канала ${ }^{1}[18,21-23]$.

Электростатические зеркала, использующие электрические поля, рассчитанные по формуле Донкина (3), обеспечивают идеальные решения для поворотных устройств. В работах $[1,2]$ для этой цели предлагалось использовать электрическое поле с потенциалом

$$
U(x, y, z)= \begin{cases}\frac{U_{0} x}{z+\sqrt{x^{2}+y^{2}+z^{2}}} & \text { при } x \geq 0, \\ 0 & \text { при } x \leq 0 .\end{cases}
$$

Координаты $x, y, z$ в этой и последующих формулах, а также на графиках, показанных на рис. 1, представляют собой безразмерные нормированные величины, так что при привязке к конкретной конструкции следует использовать подстановку $x \rightarrow x / L, y \rightarrow y / L, z \rightarrow z / L$, где $L-$ характерный масштаб конструкции. В качестве масштабирующего коэффициента для безразмерных (нормированных) потенциалов здесь и далее удобно выбирать безразмерный множитель $U_{0}=1$, который в силу этого можно не указывать явно в соответствующих формулах. Этот масштабирующий множитель не влияет на качественное поведение траекторий как единого целого и, следовательно, не может использоваться как управляющий параметр при оптимизации оптических свойств. Отметим, что при выборе нормировочного коэффициента $U_{0}=1$ значения безразмерного потенциала (4) лежат в диапазоне $[0,1)$, а предельное значение $U_{\infty}=1.0$ соответствует бесконечно удаленным точкам пространства и никогда не достигается (следовательно, электроды, соответствующие безразмерному граничному условию $U=1.0$, для данного модельного потенциала не существуют).

Эквипотенциальные линии в сечении $z=1$ и эквипотенциальные поверхности для электрического потенциала (4) показаны на рис. $1, a, b$. Трехмерные эквипотенциальные поверхности для потенциалов вида (3) представляют собой конические поверхности (вообе

\footnotetext{
${ }^{1}$ На самом деле в этом устройстве используется входной пучок, расходящийся с малым углом раствора, а не строго параллельный. Поэтому телескопическое поворотное устройство с полем $U(x, y, z) \sim \operatorname{arctg}(x / z)$ создает промежуточный мнимый источник с большими углами ввода непосредственно на входе в энергоанализирующую область.
} 

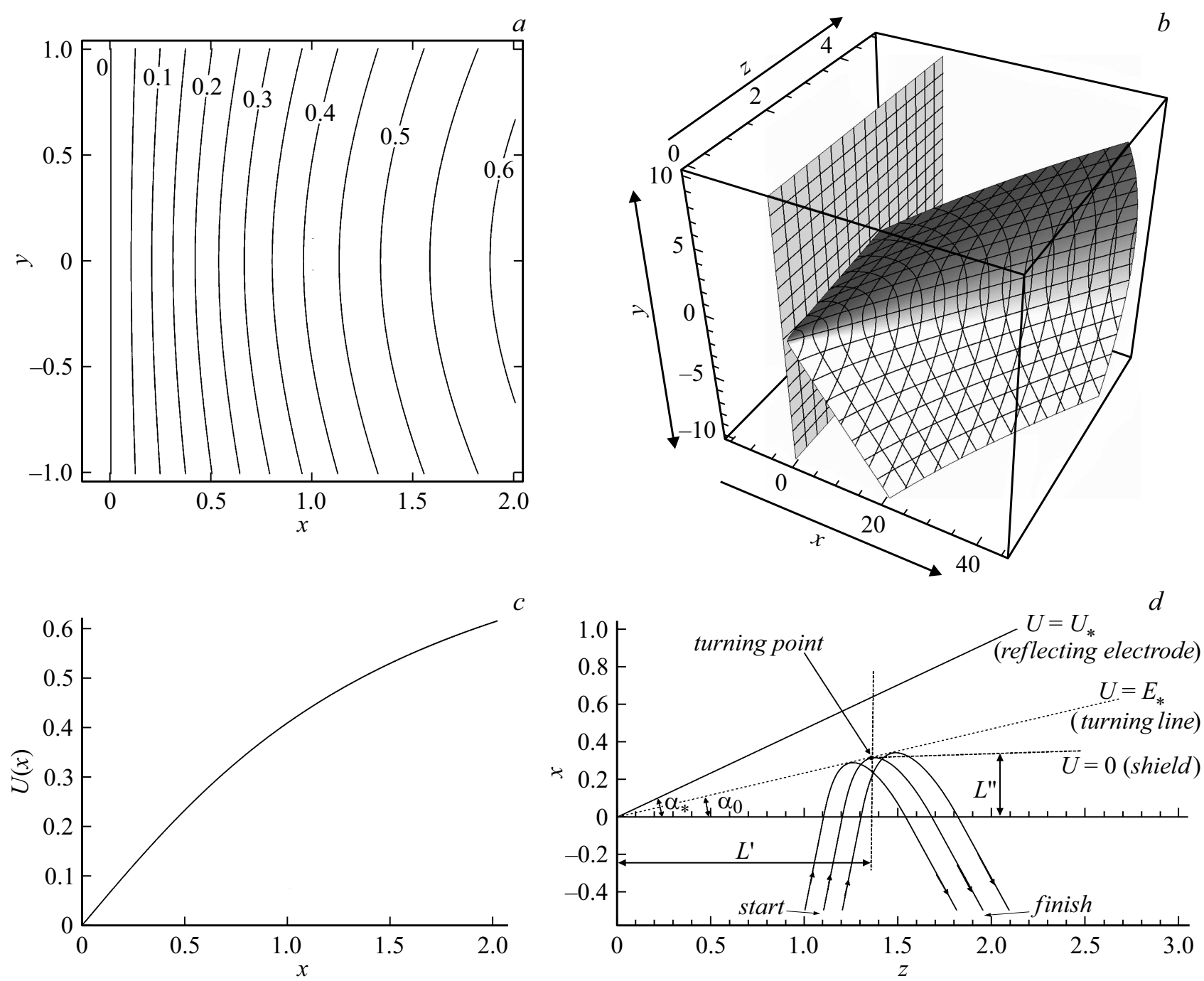

Рис. 1. Свойства безразмерного электрического потенциала (4), соответствующего значению нормировочного (масштабирующего) коэффициента $U_{0}=1: a$ - эквипотенциальные линии в сечении $O X Y$ при $x=1 ; b$ - трехмерные эквипотенциальные поверхности (полезадающие электроды) при безразмерных граничных значениях $U=0.0$ и $U=0.9$; $c-$ распределение потенциала вдоль прямой линии $z=1, y=0 ; d-$ характерные параметры для траекторий и электродов поворотной системы в сечении $O X Y$ (см. пояснения в тексте).

говоря, криволинейные) с центром в начале координат и проходящие через эквипотенциальные линии в фиксированном сечении $z=$ const. На рис. $1, c$ показан профиль электрического потенциала (4) при фиксированных значениях $z=1, y=0$ по мере углубления во внутреннюю область зеркала (т.е. при возрастании координаты $x$ ). При $U_{0}>0$ электрическое поле (4) действует на положительно заряженные частицы как зеркало.

Масштабирующие множители, выбранные для формулы (4), возможно, и отражают ее математическое изящество, но они лишь в малой степени полезны для привязки нормированных безразмерных выражений и рисунков к конкретной конструкции физического устройства. Так, множитель $U_{0}$ соответствует значению электрического потенциала $U(x, y, z)$, достигаемому на бесконечности, и поэтому его нельзя использовать ни в качестве характерной разности потенциалов между двумя полезадающими электродами устройства, ни в качестве характерной энергии отражаемого пучка заряженных частиц. Точно так же нормировочный множитель $L$, введенный формальным образом, будет не слишком полезен, поскольку выражение (4) по сути своей безразмерно. Кроме того, потенциал $U(x, y, z)$ сохраняет свое значение вдоль прямых лучей (эквипотенциальных поверхностей-электродов), проходящих через начало координат, так что, вообще говоря, электроды устройства неограниченно продолжаются на бесконечность.

Фактически при выбранной системе нормировки геометрический масштаб устройства контролируется не каким-либо характерным расстоянием между двумя полезадающими электродами, а углом раствора между ними, поэтому истинные нормирующие параметры сле- 
дует выбирать иным образом. На рис. $1, d$ показаны характерные геометрические параметры для рассматриваемой поворотной системы: $L^{\prime}-$ сдвиг по горизонтали точки поворота пучка заряженных частиц относительно условного начала координат, $L^{\prime \prime}-$ характерная глубина погружения пучка заряженных частиц в отражающее электрическое поле, $\alpha_{*}$ - угол на плоскости $O X Y$ между заземленным входным экраном и отражающим электродом, $E_{*}$ - кинетическая энергия входного пучка заряженных частиц (выраженная в электронвольтах), $U_{*}$ - потенциал на отражающем электроде.

Если ввести обозначение $\operatorname{tg}\left(\alpha_{0}\right)=L^{\prime \prime} / L^{\prime}$, то угол между полезадающими электродами должен выбираться в соответствии с условием $\operatorname{tg}\left(\alpha_{*}\right)=k \operatorname{tg}\left(\alpha_{0}\right)$, где $k \approx 1.25-1.5$ - коэффициент безопасности, определяющий расстояние от точки поворота пучка до отражающего электрода. Потенциал $U_{*}$ и энергия $E_{*}$, вычисляемые в соответствии с формулой (4), будут связаны с масштабирующим множителем $U_{0}$ соотношениями

$E_{*}=\frac{\operatorname{tg}\left(\alpha_{0}\right)}{1+\sqrt{1+\operatorname{tg}^{2}\left(\alpha_{0}\right)}} U_{0}, U_{*}=\frac{k \operatorname{tg}\left(\alpha_{0}\right)}{1+\sqrt{1+k^{2} \operatorname{tg}^{2}\left(\alpha_{0}\right)}} U_{0}$.

Из первого соотношения определяется нормирующий множитель $U_{0}$, соответствующий заданной характерной энергии пучка $E_{*}$. После этого из второго выражения можно определить напряжение $U_{*}$ для отражающего электрода. Этими вычислениями завершается привязка абстрактной безразмерной формулы (4) к реальной конструкции физического устройства с заданными ограничениями на требуемые геометрические размеры и энергии.

Аналогичный анализ потребуется при привязке конструкции физического устройства к безразмерным выражениям и нормированным рисункам для модельных потенциалов, которые приводятся далее в настоящей работе. Однако указанная система нормировки с выбором характерного размера системы как $\sim \sqrt{\left(L^{\prime}\right)^{2}+\left(L^{\prime \prime}\right)^{2}}$ имеет смысл лишь тогда, когда энергия для базовой траектории фиксирована, а интересующий исследователя пучок представляет собой набор заряженных частиц с достаточно малым разбросом по начальным углам, координатам и энергиям относительно базовой траектории. В противном случае будет некорректным использовать параметр $\sqrt{\left(L^{\prime}\right)^{2}+\left(L^{\prime \prime}\right)^{2}}$ как единицу геометрического масштабирования результатов расчета рассматриваемой оптической системы. В оправдание использованного выше подхода следует отметить, что, вообще говоря, на практике применение входных пучков с малым разбросом по энергии характерно как для поворотных систем, так и для монохроматоров: выходной угол в подобного рода оптических системах меняется при варьировании начальной кинетической энергии частиц весьма заметно, так что представляется затруднительным сфокусировать, транспортировать к точке детектирования либо еще каким-нибудь полезным образом использовать полученный на выходе устройства пучок с большим пространственно-угловым разбросом заряженных частиц.

Достоинством полевой структуры (4) является то, что ее электроды, т.е. заземленная вертикальная плоскостьсетка и выпуклый сегмент кругового конуса с приложенным к нему отражающим потенциалом, являются легко изготавливаемыми конструктивными элементами. Тонким моментом при использовании полевой структуры (4) является ее кусочно-сегментная природа. Электростатическое поле фактически распадается на два независимых полевых фрагмента, каждый из которых по отдельности является однородным по Эйлеру. Можно ли рассматривать такое составное поле в качестве поля, однородного по Эйлеру, является нетривиальным вопросом. Более подробно ограничения, возникающие в процессе синтеза электронно- и ионно-оптических конфигураций с привлечением кусочно-сегментных полевых структур, где требуется обеспечить подобие траекторий заряженных частиц как единого целого при вынесенных за пределы поля объекте исследования либо точки детектирования пучка, рассматриваются в разд. 2 .

На рис. 2, $a$ показаны траектории ионов в плоскости симметрии $O X Y$ для электростатического зеркала (4). $\mathrm{B}$ соответствии с общей теорией входной моноэнергетический пучок параллельных траекторий, двигающийся в плоскости симметрии поля, преобразуется в выходной пучок параллельных траекторий, где угол поворота и точка выхода пучка из зеркала зависит от энергии пучка, а степень сжатия пучка определяется законом подобия траекторий, т.е. их пропорциональным растяжением. Специфика использования принципа подобия траекторий в эйлеровых полях нулевой степени применительно к поворотным и призматическим оптическим устройствам по сравнению с его использованием для спектрографических оптических устройств (в частности, вынесение объекта, т. е. источника заряженных частиц, за пределы электростатического поля) более подробно рассматривается далее в разд. 3 .

К сожалению, на практике поворотная система с электрическим потенциалом (4) будет неработоспособной. Поведение пучка заряженных частиц будет неустойчивым: при малых отклонениях пучка от плоскости симметрии он быстро уходит на бесконечность (рис. $2, b-d)$. Это связано с тем, что критерий устойчивости $\left.\left(U_{x x}+U_{z z}\right)\right|_{y=0}<0$ [24], обеспечивающий устойчивость при движении заряженных частиц в окрестности плоскости симметрии $y=0$, для потенциала (4) не выполнен:

$$
\left.\left(U_{x x}+U_{z z}\right)\right|_{y=0}=\frac{U_{0} x}{\sqrt{x^{2}+z^{2}}\left(z+\sqrt{x^{2}+z^{2}}\right)^{2}}>0 .
$$

В вертикальной проекции эквипотенциальные поверхности электрического поля (4) являются выпуклыми (рис. $1, a)$ и следовательно, в случае тормозящего электрического поля дефокусирующими. В силу этого имеет место экспоненциальная неустойчивость траекторий при 

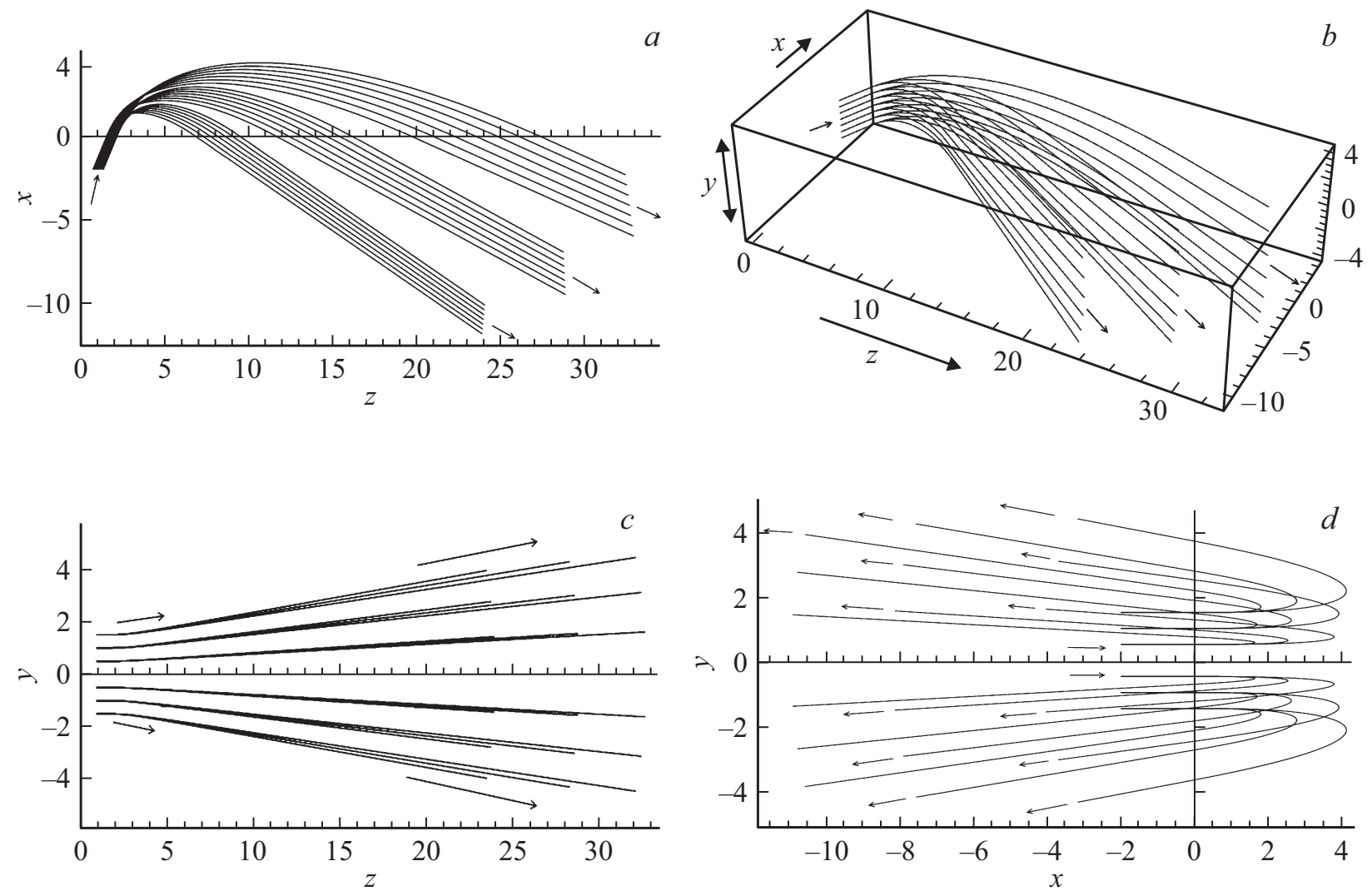

Рис. 2. Поведение траекторий заряженных частиц для электрического потенциала (4). $a$ - параллельные моноэнергетические пучка, двигающиеся в плоскости симметрии $O X Y, b$ - трехмерные траектории, смещенные в начальной точке от плоскости симметрии по координате $y, c$ - проекции трехмерных траекторий, смещенных в начальной точке от плоскости симметрии по координате $y$, на плоскость $O X Y, d$ - проекции трехмерных траекторий, смещенных в начальной точке от плоскости симметрии по координате $y$, на плоскость $O X Y$.

их отклонении от плоскости симметрии, которая наглядно иллюстрируется поведением пробных траекторий на рис. $2, b-d$.

Следовательно, для эффективной работы поворотного устройства, синтезированного с помощью формулы Донкина (3), мало обеспечить монотонный рост потенциала при углублении в область электрического поля (в противном случае устройство не сможет работать как электростатическое зеркало и не будет отражать заряженные частицы). Требуется также, чтобы был выполнен достаточный критерий устойчивости $\left.\left(U_{x x}+U_{z z}\right)\right|_{y=0}<0$ [24]. Кроме того, при использовании кусочно- сегментных полевых конфигураций, к которым относится, в частности, и электрическое поле (4), необходимо обеспечивать глобальное геометрическое подобие траекторий заряженных частиц при вынесенном за пределы поля объекте исследования либо точки детектирования пучка.

В последующих разделах рассматриваются особенности применения электрических полей с нулевой степенью однородности при синтезе поворотных и призматических электронно- и ионно-оптических систем с привлечением для этой цели формулы Донкина для однородных гармонических функций нулевой степени. Разд. 2 исследует как вопрос о технической реализуе- мости принципа подобия траекторий в случае кусочносегментных полевых конфигураций, так и общефилософский вопрос, в какой мере кусочно-сегментные полевые конфигурации допустимо называть полями, однородными по Эйлеру. Разд. 3 сравнивает между собой особенности применения принципа подобия траекторий $[16,17]$ в системах спектрографического типа (энергоспектрографов, масс-спектрографов) и в поворотных и призматических оптических элементах. Разд. 4 и 5 объясняют, как можно целенаправленно синтезировать аналитические электрические потенциалы с нулевой степенью однородности, в том числе и кусочно-сегментные, которые обладают как монотонным ростом потенциала при углублении в область электрического поля, так и устойчивостью траекторий при малых отклонениях от плоскости симметрии. В разд. 6 исследуется возможность преобразования кусочно-сегментных полевых конфигураций в конфигурации с гладко меняющимся эйлеровым полем посредством бессеточных диафрагм специфической конструкции, обеспечивающих однородность по Эйлеру для краевых полей. В разд. 7 обсуждается возможность замены криволинейных электродов сложной формы, порождаемых эквипотенциальными поверхностями аналитически заданных потенциалов, на более 
простые электроды конструктивной формы (плоскости и круговые конуса). Наконец, в разд. 8 в краткой форме приводятся результаты численного моделирования простой поворотной системы, использующей однородные по Эйлеру электростатические поля с нулевой степенью однородности. Разд. 9 посвящен заключению и выводам.

\section{2. Кусочно-сегментные электростатические структуры и принцип глобального подобия траекторий}

Одна из особенностей полевой структуры (4) состоит в том, что она составлена из двух отдельных полевых „кусков“", состыкованных вместе - электростатического зеркала с ненулевым электрическим полем и бесполевого дрейфового пространства. При этом каждый из фрагментов характеризуется своим собственным электрическим полем, однородным по Эйлеру в пределах соответствующей подобласти (электрическое поле, равное нулю, является частным случаем поля, однородного по Эйлеру). Вообще говоря, далеко не факт, что подобная кусочно-однородная полевая конфигурация (в частности, с вынесенными за пределы поля источника заряженных частиц либо точкой детектирования заряженных частиц) будет подчиняться принципу подобия траекторий, а также что такое поле допустимо называть полем, однородным по Эйлеру.

Для того чтобы принцип подобия траекторий сохранял свою силу для кусочно- сегментной полевой структуры, должны выполняться некоторые дополнительные требования:

а) центр однородности поля для каждого из фрагментов рассматриваемой полевой конфигурации является одной и той же точкой пространства;

б) степень однородности поля для каждого из полевых фрагментов является одной и той же;

в) каждый из фрагментов представляет собой трехмерную область конического типа, для которой справедливо утверждение, что когда некоторая точка $(x, y, z)$ принадлежит рассматриваемой области, то этой области принадлежат также и все точки вида $(\lambda x, \lambda y, \lambda z)$ при любых $\lambda>0$;

г) границы между фрагментами представляют собой двумерные поверхности конического типа: когда некоторая точка $(x, y, z)$ лежит на границе между двумя полевыми фрагментами, то и все точки вида $(\lambda x, \lambda y, \lambda z)$ при любых $\lambda>0$ также лежат на границе между этими полевыми фрагментами;

д) электрический потенциал на границе между отдельными фрагментами непрерывен с двух сторон от границы. В частности, из условия непрерывности потенциала на границе между фрагментами следует, что поведение потенциала вдоль разграничивающей конической поверхности описывается функцией, однородной по Эйлеру с соответствующей степенью однородности.
Коническая форма индивидуальных трехмерных областей и коническая форма разграничивающих их двумерных поверхностей является важным требованием, также как и требование непрерывности электрического потенциала при пересечении границы. В частности, отсутствие скачка потенциала (но не скачка напряженности электрического поля) при пересечении границы означает, что смещения траектории либо преломления траектории при пересечении бесконечно тонкой границы между соседними полевыми фрагментами не происходит (см. [25]) - хотя, например, кривизна траектории при пересечении границы будет испытывать скачок.

Доказательство работоспособности принципа подобия траекторий для кусочно-сегментных полевых структур, удовлетворяющих этим требованиям, выглядит следующим образом. Пусть для пробной траектории начальная точка $\left(x_{0}, y_{0}, z_{0}\right)$ выбрана в некотором полевом фрагменте, однородном по Эйлеру. Из требования конической формы для областей трехмерного пространства, содержащих отдельные полевые фрагменты, следует, что масштабированная точка $\left(\lambda_{0} x_{0}, \lambda_{0} y_{0}, \lambda_{0} z_{0}\right)$ будет принадлежать этому же полевому фрагменту.

Выберем для двух пробных траекторий начальные скорости $\left(v_{x 0}, v_{y 0}, v_{z 0}\right)$ и $\left(v_{x 0}^{\prime}, v_{y 0}^{\prime}, v_{z 0}^{\prime}\right)$, удовлетворяющие требованиям масштабирования, которые нужны для справедливости принципа подобия (см. [17]). В таком случае эти две пробные траектории будут геометрически пропорциональны друг другу (пропорциональные точки выбираются в моменты времени, синхронизированные между собой в соответствии с требованиями принципа подобия [17]) вплоть до пересечения с какой-либо границей между изолированными полевыми фрагментами. Однако пересечение первой пробной траектории с какой-либо границей между фрагментами произойдет синхронно с пересечением с той же самой границей для масштабированной пробной траектории, причем координаты, углы и кинетические энергии в точках пересечения у двух траекторий будут масштабированы сообразно принципу подобия траекторий.

В силу непрерывности электрического потенциала с двух сторон от границы при пересечении бесконечно тонкой границы между соседними полевыми фрагментами не происходит ни изменения координат, ни изменения скоростей (см. [25]), хотя ускорения, которые испытывают заряженные частицы, конечно, меняются. Поэтому и после пересечения бесконечно тонкой границы между полевыми фрагментами для начальных условий двух пробных траекторий сохраняется пропорциональность, необходимая для применения принципа подобия траекторий для движения в том полевом фрагменте, в который попали заряженные частицы. Следовательно, между исследуемыми траекториями по-прежнему будет сохраняться строгая геометрическая пропорциональность, и так будет для любого полевого фрагмента, который пересекают рассматриваемые траектории заряженных частиц. 
В конечном счете, рассматриваемые траектории окажутся глобально пропорциональными друг другу как единое целое, несмотря на то, что по факту процесс движения заряженных частиц разбивается на отдельные независимые фрагменты. Следует отметить, что это доказательство справедливо также и для кусочносегментных однородных полей с произвольной степенью однородности, если только отдельные полевые фрагменты удовлетворяют ограничениям а-д.

Легко проверить, что при выполнении требований а-д составной электрический потенциал $U(x, y, z)$ окажется функцией, однородной по Эйлеру в смысле, который придается этому термину в классическом математическом анализе $[3,4]$ (см. тождество (1)). Если же области определения фрагментов функции не будут коническими телами, а границы между отдельными фрагментами не будут коническими поверхностями, то результирующую функцию нельзя считать в глобальном смысле функцией, однородной по Эйлеру.

Например, в работе [26] для некоторых систем граница между полевой частью и дрейфовой частью не удовлетворяет „коническому“ условию $(x, y, z) \in \Omega \Rightarrow \forall \lambda>0$ : $(\lambda x, \lambda y, \lambda z) \in \Omega$, а старт пучка заряженных частиц $\mathrm{c}$ разными начальными кинетическими энергиями начинается со смещением относительно начала координат (в силу чего в [26] потребовалась разработать отдельную теорию, описывающую поведение энергоанализирующих устройств). При этом, вообще говоря, нет необходимости, чтобы потенциал был непрерывен на границе между полевыми фрагментами, имеющими коническую форму - достаточно, если поведение электрического потенциала вдоль граничной поверхности представляет собой функцию, однородную по Эйлеру. Однако непрерывность электрического потенциала на границах является важным фактором, обеспечивающим соблюдение принципа подобия траекторий при движении заряженных частиц в кусочно-сегментных электростатических структуpax, являющихся однородными по Эйлеру ${ }^{2}$. Отметим что как условие непрерывности электрического потенциала на границах между фрагментами, так и условие „коничности“ $(x, y, z) \in \Omega \Rightarrow \forall \lambda>0:(\lambda x, \lambda y, \lambda z) \in \Omega$, которое должно выполняться для отдельных полевых фрагментов и для границ между ними, требуется обеспечить лишь для тех областей пространства, через которые реально проходят траектории заряженных частиц.

Можно убедиться, что, например, полевая структура (4) указанным условиям удовлетворяет. Действительно, если для точки $(x, y, z)$ выполнено условие $x>0$, то и для точки $(\lambda x, \lambda y, \lambda z)$ при $\lambda>0$ выполнено условие

\footnotetext{
2 Аккуратный анализ показывает, что с формальной точки зрения и для принципа подобия непрерывность электрического потенциала на границе тоже не требуется. И справа, и слева от границы потенциал будет однородной по Эйлеру функцией. Так что при пересчете скоростей заряженных частиц, находящихся в нужном пропорциональном отношении, после пересечения ими бесконечно тонкой границы со скачком электрического потенциала (см. [25]), скорости и координаты окажутся именно в том пропорциональном отношении, который требуется для подобия очередных фрагментов траекторий.
}

$\lambda x>0$. Точно также, если точка $(x, y, z)$ удовлетворяет условию $x<0$, то и точка $(\lambda x, \lambda y, \lambda z)$ при $\lambda>0$ удовлетворяет условию $\lambda x<0$. Наконец, если точка принадлежит плоскости $x=0$, то и масштабированные точки $(\lambda x, \lambda y, \lambda z)$ также принадлежат плоскости $x=0$. Очевидно, что в каждой из подобластей электрический потенциал (4) представляет собой однородную по Эйлеру функцию нулевой степени, поскольку $U(x, y, z)=0$ также является функцией, однородной по Эйлеру, хотя и вырожденной. Наконец, при приближении к границе $x=0$ электрический потенциал непрерывен как при $x \rightarrow 0^{+}$, так и при $x \rightarrow 0^{-}$, а на самой границе представляет собой однородную по Эйлеру функцию нулевой степени (тождественно равную нулю).

Следовательно, для движения заряженных частиц в электрическом поле с потенциалом (4) принцип подобия траекторий выполняется глобально, несмотря на то, что часть траектории (объект исследования и изображение объекта) находится в бесполевом дрейфовом пространстве, вынесенном за пределы поля. То, что получающиеся на выходе траектории действительно глобально подобны друг другу как единое целое, иллюстрируется, например, траекториями, показанными рис. 1,e, 2, $a$.

\section{3. Специфика применения принципа подобия траекторий в поворотных и призматических системах}

Сам по себе принцип подобия траекторий в полях, однородных по Эйлеру $[16,17]$, не зависит как от степени однородности поля, так и от начальных условий старта или детектирования заряженных частиц. Однако его использование при синтезе спектрографических устройств $[18,19]$ с однородными по Эйлеру полями, обладающими ненулевой степенью однородности [27], несколько отличается использования этого же принципа при синтезе поворотных $[1,2]$ и призматических [20] устройств с однородными по Эйлеру полями нулевой степени. На этом моменте стоит остановиться подробнее.

Рассмотрим движение заряженных частиц в эйлеровом поле ненулевой степени $n$. При масштабировании начальных координат в $\lambda$ раз, сохранении неизменными начальных углов и масштабировании начальной кинетической энергии в $\lambda^{n}$ раз вся траектория заряженной частицы масштабируется геометрически в $\lambda$ раз как единое целое. Если точка старта расположена в начале координат, то после масштабирования она и останется в начале координат. Масштабирование начальной энергии в $\mu$ раз (где $\mu-$ произвольное число) с точки зрения принципа подобия траекторий соответствует геометрическому масштабированию в $\lambda=\mu^{1 / n}$ раз.

В соответствии с принципом подобия если расходящиеся из начала координат под заданным начальным углом траектории с фиксированной начальной энергией фокусируются по углу в некоторой точке $\left(x_{f}, y_{f}, z_{f}\right)$, то 
в соответствии с принципом подобия траектории, характеризуемые масштабированной в $\mu$ начальной энергией и расходящиеся из того же самого начала координат под тем же самым начальным углом, сфокусируются по углу в точке с координатами $\left(\mu^{1 / n} x_{f}, \mu^{1 / n} y_{f}, \mu^{1 / n} z_{f}\right)$. При этом порядок фокусировки сохранится, а точки фокусировки по углу, соответствующие разным начальным энергиям, будут образовывать прямую линию, проходящую через начало координат. В результате такая электростатическая система оказывается идеальным энергоспектрографом, хотя, конечно, проблемы старта заряженных частиц строго из точки начала координат, где имеется особая точка электрического поля, а две эквипотенциальные поверхности, используемые как полезадающие электроды, образуют острый угол, привносит в работу рассматриваемой энергоанализирующей системы дополнительные искажения.

При движении заряженных частиц в однородном электростатическом поле нулевой степени ситуация другая. При масштабировании начальных координат в $\lambda$ раз, сохранении неизменными начальных углов и сохранении начальной кинетической энергии вся траектория заряженной частицы масштабируется геометрически в $\lambda$ раз как единое целое. Рассмотрим прямую линию, проходящую через начало координат. Выберем на этой прямой плоскую площадку с центром в точке $\left(x_{0}, y_{0}, z_{0}\right)$ и стартуем с нее под заданным начальным углом и с заданной начальной кинетической энергией пучок заряженных частиц с пространственно распределенными начальными координатами $\left(\lambda x_{0}, \lambda y_{0}, \lambda z_{0}\right)$, где $\lambda \in[1-\varepsilon, 1+\varepsilon]$. Тогда в соответствии с принципом подобия в силу правильного масштабирования начальных условий такие частицы будут двигаться по геометрически подобным траекториям, масштабированным в $\lambda$ раз.

В частном случае, когда начальный участок и конечный участок траекторий расположены в бесполевом пространстве (которое так же может рассматриваться как частный случай поля, однородного по Эйлеру), будет происходить преобразование входного параллельного монохроматического пучка траекторий в выходной параллельный монохроматический пучок траекторий. Кроме того, при старте и при детектировании траекторий в бесполевом пространстве нет необходимости располагать как точки старта, так и точки детектирования параллельного пучка непременно вдоль прямой линии (участка плоскости), проходящей через начало координат. Конечно, угол поворота пучка траекторий и степень сжатия параллельных траекторий зависят от начальной кинетической энергии и от угла влета, но строгая параллельность траекторий на входе и на выходе гарантируется (при смещении параллельных траекторий друг относительно друга в горизонтальном направлении).

Таким образом, при использовании однородных электростатических полей нулевой степени получаем идеальную электростатическую призму [20] в случае, когда угол, на который изменяется направление движения параллельного моноэнергетического пучка, достаточно мал, и идеальную поворотную (отражающую) систему, когда угол поворота приближается к $180^{\circ}$. Возможность размещать точку старта траекторий в произвольной точке пространства, а не только в начале координат, где, вообще говоря, имеется сингулярность электрического поля, является принципиальным отличием поворотных и призматических устройств от спектрографических энергоанализирующих устройств. Однако подобно тому, как для спектрографических энергоанализаторов разброс по координатам для реальных точек старта по отношению к точному началу координат приводит к ухудшению идеальных характеристик устройства, так и разброс входного параллельного монохроматического пучка по энергии и углам ухудшает идеальную работу поворотных либо призматических электростатических систем, использующих однородные электрические поля нулевой степени.

\section{4. Электростатические зеркала с гарантированной устойчивостью пучков в окрестности плоскости симметрии}

Для того чтобы для электрического потенциала вида (3) в полуплоскости $x \geq 0$ на плоскости $O X Z$ было выполнено достаточное условие устойчивости $U_{x x}+U_{z z}<-\varepsilon^{2}<0$, необходимо и достаточно, чтобы функция $f(p)=F(p, 0)$ удовлетворяла дифференциальному уравнению

$$
\frac{d^{2} f}{d p^{2}}+\frac{d f}{d p} \frac{2 p}{1+p^{2}}+\frac{g(p)}{1+p^{2}}=0
$$

где $g(p) \geq \varepsilon^{2}>0-$ произвольная функция, положительная при $p>0$. Уравнение (5) является результатом прямой подстановки выражения (3) в условие устойчивости с последующим использованием условия, что функция $F$ - гармоническая. Общее решение уравнения (5) имеет вид

$$
\begin{gathered}
f(p)=A+B \operatorname{arctg}(p)+\int_{0}^{p} \operatorname{arctg}(\xi) g(\xi) d \xi \\
-\operatorname{arctg}(p) \int_{0}^{p} g(\xi) d \xi,
\end{gathered}
$$

где свободные константы $A$ и $B$ должны выбираться в соответствии с начальными условиями $f(0)=A$, $f^{\prime}(0)=B$, задающими поведение электрического потенциала на входной границе электростатического зеркала.

При смещении потенциала на константу и при умножении потенциала на положительную константу оптические свойства электрического поля не меняются (при условии одновременного масштабирования начальной 
кинетической энергии заряженных частиц). Без ограничения общности можно считать, что $f(0)=0$ (потенциал на входе в зеркало равен нулю) и $f^{\prime}(0)=2$ (скорость роста потенциала в точке входа в зеркало фиксирована и положительна, ${ }^{3}$ значение константы выбрано из соображений удобства нормировки).

Как только задана положительная функция $g(p)$, функция $f(p)$ определяется однозначным образом в соответствии с формулой (6). Но если определена функция $f(p)$, то с помощью перехода от вещественной оси к комплексной плоскости (см. [14]) однозначным образом определяется также и функция $F(p, q)$, симметричная по переменной $q: F(p, q)=\operatorname{Re} f(p+i q)$. При известной функции $F(p, q)$ по формуле (3) можно восстановить во всем пространстве трехмерный электростатический потенциал Донкина, управляющий работой поворотного устройства. При этом существует достаточно обширное семейство функций $g(p)$, для которых выражение (6) может быть проинтегрировано в аналитической форме. В частности, выражение (6) интегрируется в аналитическом виде, если $g(p)$ - дробно-рациональная функция. Как правило, используемая для синтеза электростатического зеркала функция $g(p)$ зависит от одного или нескольких свободных параметров, изменяя которые можно управлять видом электрического потенциала поворотного устройства и формой его электродов.

Формула (6) обеспечивает выполнение критерия устойчивости $U_{x x}+U_{z z}<-\varepsilon^{2}<0$, но не гарантирует монотонного роста трехмерного потенциала при углублении в электростатическое зеркало. С учетом (3) гарантированная монотонность потенциала Донкина в плоскости симметрии $O X Z$ по координате $x$ обеспечивается при условии монотонного роста функции $f(p)$ на интервале $0 \leq p \leq 1$. Монотонный рост функции $f(p)$ в окрестности точки $p=0$ обеспечивается выбранными начальными условиями $f(0)=0, f^{\prime}(0)=2$, так что условие гарантированной монотонности сводится к тому, что в точке $p=1$ производная $f^{\prime}(p)$ неотрицательна, причем других точек на интервале $0 \leq p \leq 1$, в которых $f^{\prime}(p)$ обращается в нуль и меняет свой знак, не имеется. Проверка выполнения этого условия позволяет выявлять безопасные диапазоны изменения свободных параметров, управляющих видом функции $g(p)$.

Ниже приводятся примеры некоторых аналитических потенциалов для электростатических зеркал Донкина, которые синтезируются указанным способом. Численная проверка показывает, что для всех этих потенциалов имеет место устойчивость (фокусировка) монохроматических пучков траекторий при отклонении начальных условий (координат и углов) от плоскости симметрии. Для экономии места рисунки, аналогичные рис. 2, здесь не приводятся.

\footnotetext{
${ }^{3}$ Вырожденный случай $f^{\prime}(0)=0$ также мог бы представлять определенный интерес, но его анализ выходит за рамки данной работы.
}

Пример 1. Пусть $g(p)=a, a>0$. Решением уравнения (5) будет функция

$$
f(p)=2 \operatorname{arctg}(p)-\frac{a}{2} \ln \left(1+p^{2}\right),
$$

a ее продолжением на комплексную плоскость будет функция

$$
\begin{aligned}
& f(p+i q)=2 \operatorname{arctg}(p+i q)-\frac{a}{2} \ln \left(1+(p+i q)^{2}\right) \\
& =\operatorname{arctg}\left(\frac{2 p}{1-p^{2}-q^{2}}\right)+\frac{i}{2} \ln \left(\frac{p^{2}+(1+q)^{2}}{p^{2}+(1-q)^{2}}\right) \\
& \quad-\frac{a}{4} \ln \left(\left(1+p^{2}-q^{2}\right)^{2}+(2 p q)^{2}\right) \\
& -i \frac{a}{2} \operatorname{arctg}\left(\frac{2 p q}{1+p^{2}-q^{2}}\right) .
\end{aligned}
$$

В результате окончательным решением поставленной задачи будет функция

$$
\begin{aligned}
F(p, q)= & \operatorname{arctg}\left(\frac{2 p}{1-p^{2}-q^{2}}\right) \\
& -\frac{a}{4} \ln \left(\left(1+p^{2}-q^{2}\right)^{2}+(2 p q)^{2}\right),
\end{aligned}
$$

где для гарантированной монотонности электрического потенциала должно быть выполнено условие $0 \leq a \leq 2$. На рис. 3, a показаны сечения трехмерных эквипотенциальных поверхностей в плоскости $z=1$ для электрического потенциала, сконструированного с помощью функции (7) при $a=1$.

Пример 2. Пусть $g(p)=a\left(1+p^{2}\right)$. Решением уравнения (5) будет функция

$$
f(p)=2 \operatorname{arctg}(p)-\frac{a}{6}\left(p^{2}+2 \ln \left(1+p^{2}\right)\right) .
$$

После продолжения на комплексную плоскость ей соответствует гармоническая функция двух переменных

$$
\begin{aligned}
& F(p, q)=\operatorname{arctg}\left(\frac{2 p}{1-p^{2}-q^{2}}\right) \\
& -\frac{a}{6}\left[p^{2}-q^{2}+\ln \left(\left(1+p^{2}-q^{2}\right)^{2}+(2 p q)^{2}\right)\right] .
\end{aligned}
$$

Для гарантированной монотонности соответствующего электрического потенциала должно быть выполнено условие $0 \leq a \leq 3 / 2$. На рис. $3, b$ показаны сечения трехмерных эквипотенциальных поверхностей в плоскости $z=1$ для электрического потенциала, сконструированного с помощью функции (8) при $a=1 / 2$.

Пример 3. Возьмем $g(p)=a \frac{\left(1+p^{2}\right)}{\left(1-p^{2}\right)^{2}}$. Решением уравнения (5) будет функция

$$
f(p)=2 \operatorname{arctg}(p)+\frac{a}{4} \ln \left(\frac{1-p^{2}}{1+p^{2}}\right),
$$



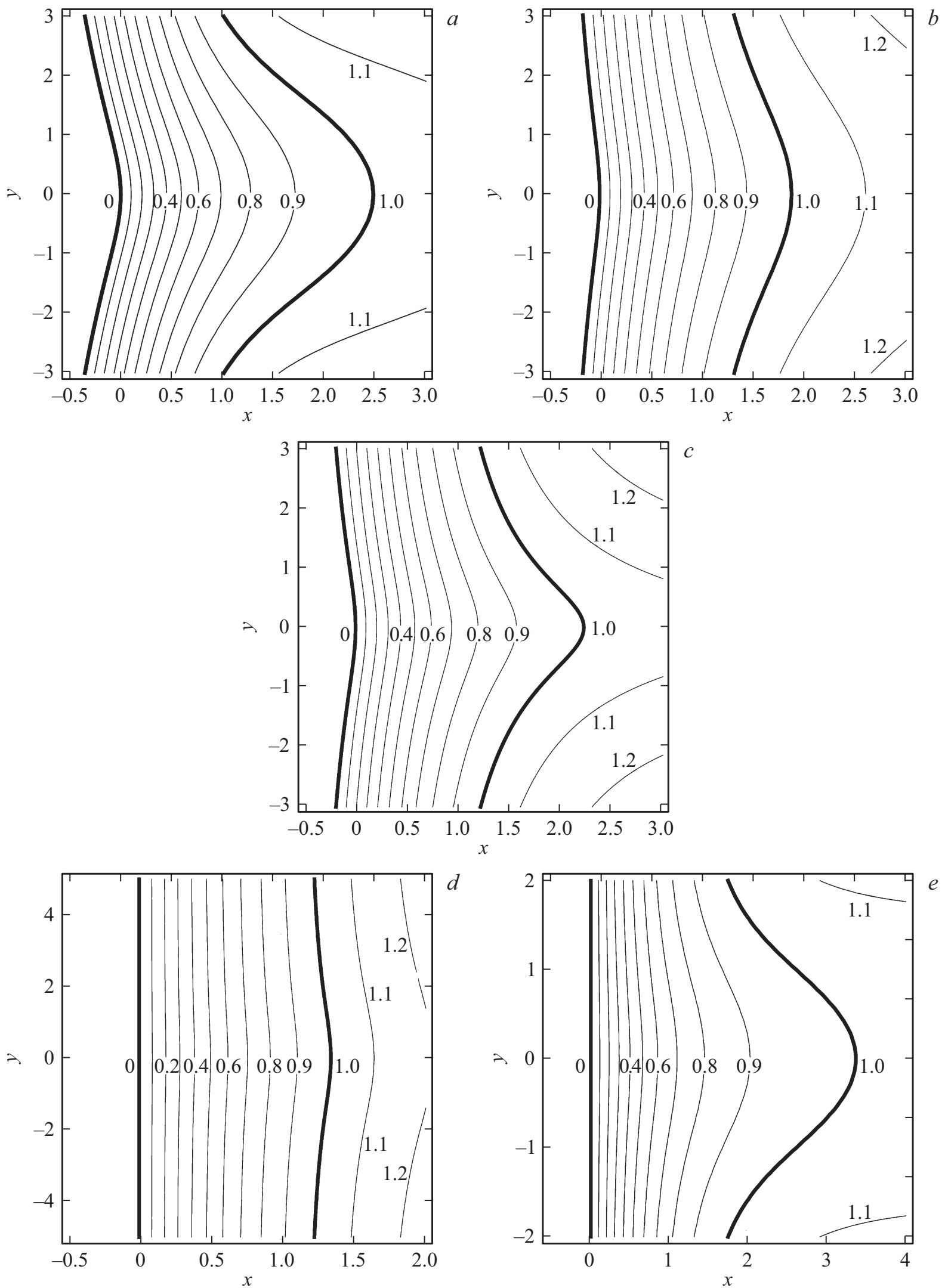

Рис. 3. Эквипотенциальные линии в сечении $x=1$ для электрических потенциалов Донкина, сконструированных с помощью: $a-$ функции (7) при $a=1, b-$ функции (8) при $a=1 / 2, c-$ функции (9) при $a=2.3, d-$ функции (10) при $a=2 / 3$, $e-$ функции (11) при $a=1$. Жирными линиями выделены эквипотенциальные линии, которые могут использоваться в качестве полезадающих электродов. 
откуда следует, что

$$
\begin{aligned}
F(p, q)= & \operatorname{arctg}\left(\frac{2 p}{1-p^{2}-q^{2}}\right) \\
& +\frac{a}{8} \ln \left(\frac{p^{4}-2 p^{2}\left(1-q^{2}\right)+\left(1+q^{2}\right)^{2}}{p^{4}+2 p^{2}\left(1+q^{2}\right)+\left(1-q^{2}\right)^{2}}\right)
\end{aligned}
$$

Гарантированная монотонность электрического потенциала (9), т. е. монотонность функции $f(p)$ на всем диапазоне значений $0 \leq p \leq 1$, не может быть достигнута. Однако гарантированная монотонность потенциала не самоцель. Для функционирования зеркала достаточно, чтобы функция $f(p)$ монотонно росла в некотором диапазоне значений $0 \leq p \leq p_{0}$. Например, для выполнения условия монотонности в диапазоне $0 \leq p \leq 2 / 3$ достаточно условия $0 \leq a \leq 5 / 3$. На рис. $3, c$ показаны сечения трехмерных эквипотенциальных поверхностей в плоскости $z=1$ для электрического потенциала, сконструированного с помощью функции (9) при $a=2 / 3$.

\section{5. Электростатические зеркала с плоским входным электродом}

Представляется удобным, если входной электрод поворотной системы будет вертикальной плоскостью (рис. $1, b)$. Этого можно добиться, если использовать в формуле (6) функции $g(p)$ специального вида.

Чтобы потенциал (3) принимал нулевое значение вдоль вертикальной плоскости $x=0$, необходимо и достаточно, чтобы функция $F(p, q)$ обращалась в нуль вдоль линии $p=0$. Отсюда следует, что гармоническая функция $F(p, q)$, рассматриваемая на всей плоскости $(p, q)$, должна быть антисимметричной функцией от переменной $p: F(-p, q)=-F(p, q)$. Это означает, что функция $f(p)=F(p, 0)$ тоже является антисимметричной функцией от переменной $p: f(-p)=-f(p)$.

Данное рассуждение работает и в обратном порядке. Если $f(p)$ будет антисимметричной функцией от переменной $p$, то гармоническая функция $F(p, q)$ тоже будет антисимметричной функцией от переменной $p$ (как продукт аналитического продолжения с вещественной оси на всю комплексную плоскость для функции $f(p)$, которая содержит лишь нечетные степени $p$ при разложении ее в ряд Тейлора в точке $p=0$ ). Тогда потенциал (3) будет антисимметричной функцией по переменной $x$, а вертикальная плоскость $x=0$ будет эквипотенциальной поверхностью с нулевым значением потенциала.

Итак, для того чтобы потенциал (3) принимал нулевое значение вдоль вертикальной плоскости $x=0$, необходимо и достаточно, чтобы функция $f(p)$ была антисимметричной (нечетной). Из выражения (6) следует, что это условие выполнено, если функция $g(p)$ будет нечетной. Действительно: а) $A+B \operatorname{arctg}(p)$ будет нечетной функцией в силу условия $f(0)=0$, б) функция $\operatorname{arctg}(p) g(p)$ будет четной функцией, и поэтому интеграл от нее будет нечетной функцией, в) интеграл от нечетной функции $g(p)$ будет четной функцией, а результат его умножения на $\operatorname{arctg}(p)$ будет нечетной функцией, г) сумма трех нечетных функций будет нечетной функцией. Следовательно, если в формуле (6) выбирать в качестве функции $g(p)$ нечетную функцию, то потенциал Донкина (3) будет принимать нулевое значение вдоль вертикальной плоскости $x=0$.

Ниже рассматриваются соответствующие примеры. Численная проверка показывает, что, как и следовало ожидать, для указанных потенциалов имеет место устойчивость (фокусировка) монохроматических параллельных пучков траекторий при их отклонении от плоскости симметрии системы, а входной электрод во всех случаях представляет собой вертикальную плоскость.

Пример 4. Возьмем $g(p)=a p$. Решением уравнения (5) будет функция

$$
f(p)=2 \operatorname{arctg}(p)+\frac{a}{2}(\operatorname{arctg}(p)-p),
$$

откуда следует, что

$$
\begin{aligned}
F(p, q)= & \operatorname{arctg}\left(\frac{2 p}{1-p^{2}-q^{2}}\right) \\
& +\frac{a}{4}\left(\operatorname{arctg}\left(\frac{2 p}{1-p^{2}-q^{2}}\right)-2 p\right) .
\end{aligned}
$$

Гарантированная монотонность соответствующего электрического потенциала достигается при любых значениях параметра $a$. На рис. $3, d$ показаны сечения трехмерных эквипотенциальных поверхностей в плоскости $z=1$ для электрического потенциала, сконструированного с помощью функции (10) при $a=2 / 3$.

Пример 5. Возьмем $g(p)=a p\left(1+p^{2}\right)$. Решением уравнения (5) будет функция

$$
f(p)=2 \operatorname{arctg}(p)+\frac{a}{4}\left(\operatorname{arctg}(p)-p-\frac{1}{3} p^{3}\right),
$$

откуда следует, что

$$
\begin{aligned}
& F(p, q)=\operatorname{arctg}\left(\frac{2 p}{1-p^{2}-q^{2}}\right) \\
& +\frac{a}{8}\left(\operatorname{arctg}\left(\frac{2 p}{1-p^{2}-q^{2}}\right)-4\left(p-p q^{2}+\frac{1}{3} p^{3}\right)\right) .
\end{aligned}
$$

Гарантированная монотонность соответствующего электрического потенциала достигается при $0 \leq a \leq 8 / 7$. На рис. 3, $a$ показаны сечения трехмерных эквипотенциальных поверхностей в плоскости $z=1$ для электрического потенциала, сконструированного с помощью функции (11) при $a=1$.

\section{6. Бессеточные диафрагмы и краевые поля, однородные по Эйлеру}

Достоинством потенциалов, однородных по Эйлеру, которые были рассмотрены в предыдущих разделах, 
является то, что они представлены в простой аналитической форме, включающей в себя нескольких свободных параметров. Это позволяет достаточно просто исследовать и оптимизировать оптические свойства соответствующих систем.

Однако в этом случае серьезной проблемой будет организация ввода и вывода заряженных частиц. В рассмотренных в предыдущем разделе примерах заряженные частицы должны входить в электрическое поле и выходить из электрического поля сквозь заземленный бесконечно тонкий электрод, предполагая, что этот электрод представляет собой идеальную сетку. Реальная сетка, однако, серьезно ухудшит характеристики поворотного устройства, начиная с пропускания.

Было бы удобно, если ввод и вывод можно выполнить через специальным образом организованную бессеточную диафрагму. Однако образующиеся в этом случае паразитные краевые поля не просто возмущают движение заряженных частиц. Скорее всего, полученное электрическое поле вообще потеряет свое свойство быть однородным по Эйлеру. Это означает, что базовое свойство преобразовывать входные параллельные пучки в выходные параллельные пучки у такого поворотного устройства также будет утрачено, так как появятся паразитные коэффициенты аберрационного разложения траектории, обусловленные воздействием краевого поля.

С данной проблемой позволяет справиться алгоритм синтеза краевых полей, однородных по Эйлеру, который рассматривается в [28,29]. В этом случае исходный электрический потенциал для полупространства $z>0$ записывается с помощью взаимно-однозначной замены переменных Донкина в координатах $(p, q, r)$ :

$$
\left\{\begin{array} { l } 
{ p = \frac { x } { z + \sqrt { x ^ { 2 } + y ^ { 2 } + z ^ { 2 } } } , } \\
{ q = \frac { y } { z + \sqrt { x ^ { 2 } + y ^ { 2 } + z ^ { 2 } } } , } \\
{ r = z + \sqrt { x ^ { 2 } + y ^ { 2 } + z ^ { 2 } } , }
\end{array} \Leftrightarrow \left\{\begin{array}{l}
x=p r, \\
y=q r, \\
z=\frac{1-p^{2}-q^{2}}{2} r,
\end{array}\right.\right.
$$

а уравнение Лапласа преобразуется в эквивалентное эллиптическое уравнение в частных производных.

Переменная $r$ в полученное в конечном итоге уравнение не входит из-за однородности трехмерного потенциала и поэтому выпадает из рассмотрения. Поэтому краевая задача для трехмерного уравнения Лапласа с поверхностями-электродами и краевыми условиями Дирихле преобразуется в двумерную краевую задачу для двумерного уравнения Лапласа, определенную на плоскости $(p, q)$. Для этой задачи границами с заданными краевыми условиями Дирихле будут криволинейные линии, представляющие собой образы электродов после преобразования (12).

Как и исходные электроды, эти новые границы будут сплошными. Однако если проделать в двумерной границе, соответствующей заземленному электроду, дополнительную ,дырку“ и решить эту краевую задачу (аналитически или численно), то после обратного перехода от координат $(p, q, r)$ к координатам $(x, y, z)$ получим новый трехмерный электростатический потенциал, также однородный по Эйлеру с нулевой степенью однородности. Образ границы с „дыркой“ превратится в электрод с профилированной специальным образом бессеточной диафрагмой, так что новое электрическое поле изменится, по крайней мере, для краевой области. Однако важно, что при использовании таких специальным образом синтезированных бессеточных диафрагм трехмерное электрическое поле сохранит свойство однородности по Эйлеру во всех своих точках, включая сюда краевую область.

Если „дырка“ достаточно мала по сравнению с типичными расстояниями между краевыми границами, то она не будет сильно искажать решение двумерного уравнения Лапласа. Поэтому для новой электродной конфигурации искажению подвергнуться лишь сама бессеточная диафрагма и создаваемое ею краевое поле. Остальные же электроды, расположенные достаточно далеко от „дырки“, сохранят свою прежнюю форму, точно так же как и электрическое поле в основном объеме устройства вдали от привнесенных краевых полей практически останется прежним.

Для электродов, соответствующих нулевому значению однородного по Эйлеру потенциала, синтезируемая бессеточная диафрагма обязательно будет иметь вид клиновидной щели $[28,29]$. Однако для полей, однородных по Эйлеру с нулевой степенью однородности, любые ,дырки“ при обратном переходе к трехмерному пространству превращаются в клиновидные щели соответствующего размера, проделанные в криволинейном электроде конической формы.

Даже если добавленная к заземленному электроду клиновидная щель будет достаточно узкой по сравнению с расстоянием между электродами, ее наличие все равно сместит угол поворота параллельного пучка и изменит основные оптические характеристики устройства. Однако при этом базовое свойство поворотной системы преобразовывать входные параллельные монохроматические пучки в выходные параллельные монохроматические пучки останется в неприкосновенности. Поэтому можно просто снабдить заземленный электрод клиновидным разрезом и исследовать оптические свойства получившейся электродной структуры (вероятно, численно), не прибегая к сложному алгоритму синтеза краевых полей, описанному в [28,29].

\section{7. Использование электродов конструктивной формы}

Серьезным недостатком систем с аналитически заданными потенциалами является то, что соответствующие этим потенциалам электроды имеют сложную геометрическую форму, не слишком конструктивную с точки зрения изготовления реальных устройств. Например, среди 


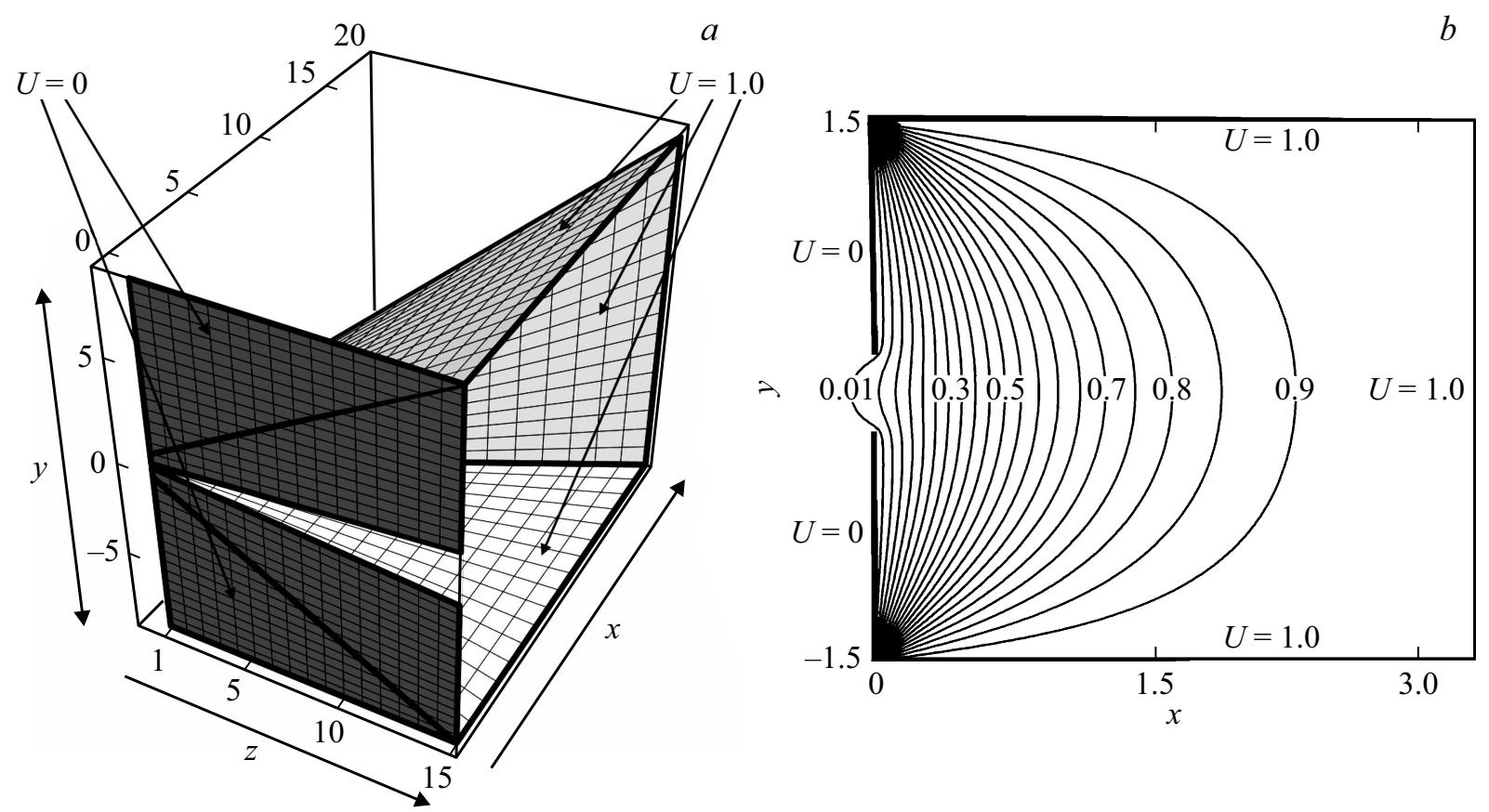

Рис. 4. Поворотная система, составленная из вертикальной заземленной плоскости с клиновидной щелью и отражающего электрода с единичным потенциалом, имеющего форму половины пирамиды с прямоугольным основанием и осью, совпадающей с координатной осью $O Z: a$ - трехмерное расположение электродов, $b-$ эквипотенциальные линии численно рассчитанного электрического поля в плоскости $z=5$. (Все координаты и потенциалы указаны здесь в безразмерных нормированных величинах.)

рассмотренных выше электродов только эквипотенциальные поверхности в виде заземленной плоскости $x=0$ могут рассматриваться как простые в изготовлении.

В качестве конструктивных электродов при создании устройств подобного вида разумно ограничиться электродами, представляющими собой фрагменты плоскостей и круговых конусов (возможно, с клиновидными вырезами, проходящими через начало координат). Специфика потенциалов, однородных по Эйлеру с нулевой степенью однородности, состоит в том, что тот же самый подход, который применяется для синтеза однородных краевых полей, позволяет справиться и с проблемой неконструктивных электродов. (Для потенциалов, однородных по Эйлеру с ненулевыми степенями однородности, это не так.) Рассмотрим этот процесс подробнее.

Достаточно трудоемкий, но не представляющий особых технических проблем, анализ показывает, что после выполнения замены переменных (12) „отпечатками“ на плоскости $(p, q)$ для плоскостей и круговых конусов с центром в начале координат и их конических фрагментов будут отрезки прямых и дуги окружностей. Стоит при этом отметить, что отпечатками конических сегментов круговых конусов могут быть отрезки прямых, а отпечатками клиновидных сегментов плоскостей - дуги окружностей. Справедливо и обратное утверждение: кривые на плоскости $(p, q)$, являющиеся сегментами прямых и дугами окружностей, при обратном переходе к трехмерному пространству с помощью подстановки (12) превращаются в конические сегменты круговых конусов и/или в клиновидные сегменты плоскостей. Поэтому если выбрать на плоскости $(p, q)$ границы в виде отрезков прямых и дуг окружностей и решить соответствующую краевую задачу, мы не только получим после замены переменных (12) некоторое трехмерное электрическое поле нулевой степени, но и гарантируем конструктивность трехмерных электродов, с помощью которых создается это электрическое поле.

При некоторой удаче с помощью общих методов теории функций комплексного переменного [30,31] и справочников по конформным отображениям [32] для определенных краевых задач с прямолинейными отрезками и дугами окружностей в качестве границ можно найти аналитическое решение. Можно также воспользоваться для этой цели численными методами построения конформных отображений [33], получив на выходе аналитическое выражение с подставленными в него приближенными числовыми параметрами. Но все же кроме отдельных случаев специального вида у краевой задачи не удается найти решение в аналитической форме, и поэтому в большинстве своем эти задачи приходится рассчитывать численно.

Еще раз подчеркнем важный вывод: численное или аналитическое решение на плоскости $(p, q)$ краевой задачи с границами в виде отрезков прямых и дуг окружностей порождает в пространстве $(x, y, z)$ трехмерный потенциал $V(x, y, z)$ с конструктивными электродами в виде сегментов круговых конусов и плоскостей. Это позволяет значительно упростить работу исследователя 

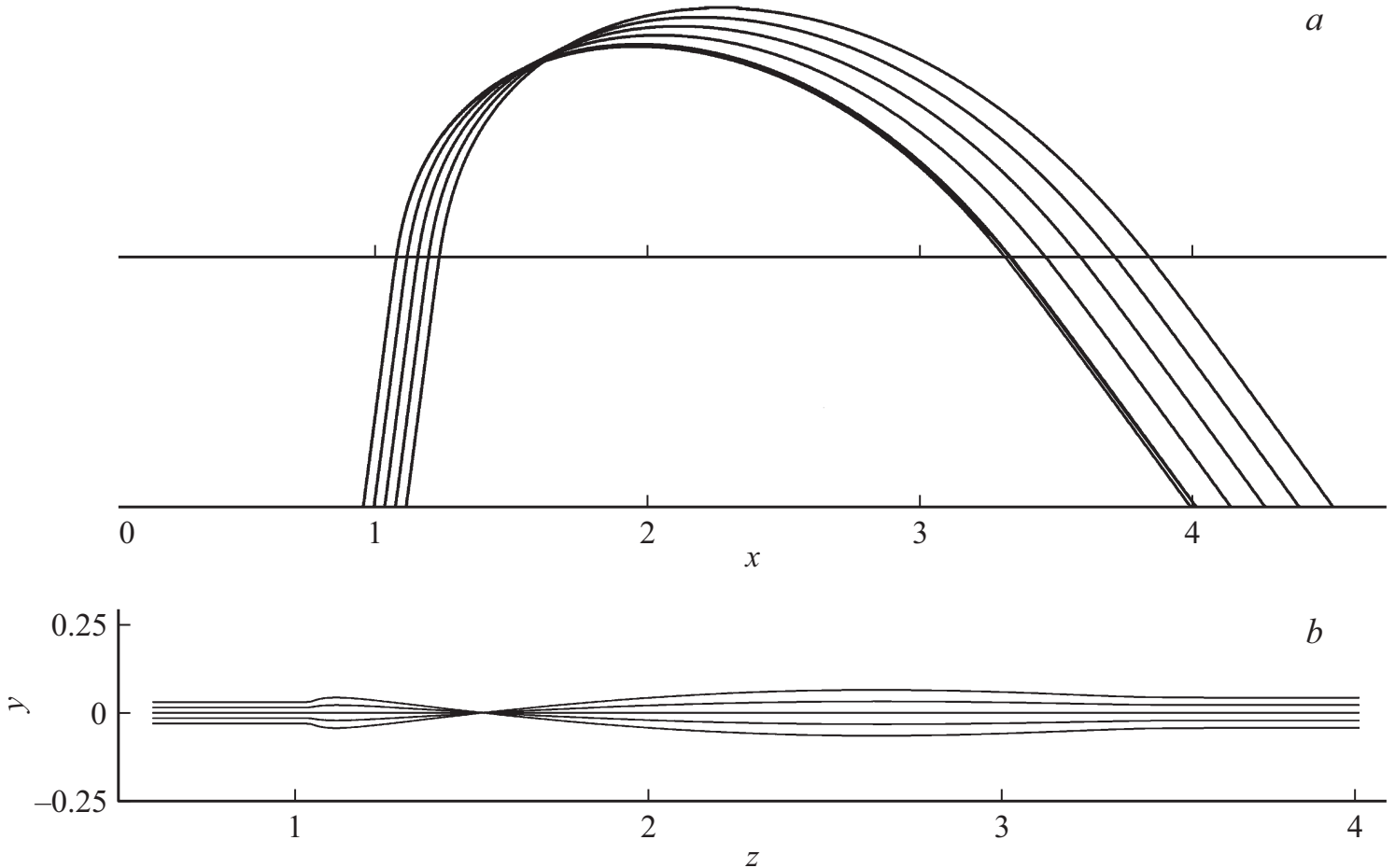

Рис. 5. Траектории для поворотной системы на рис. 4: $a$ - горизонтальная проекция на плоскость симметрии для параллельного пучка траекторий с начальным разбросом по горизонтальной координате, $b-$ вертикальная проекция для параллельного входного пучка с начальным разбросом по вертикальной координате (телескопический режим вертикальной фокусировки).

при конструировании с помощью формулы Донкина поворотных устройств, задача которых состоит в преобразовании входных параллельных монохроматических пучков в выходные параллельные монохроматические пучки.

Строго говоря, нет необходимости вообще пользоваться формулой Донкина, раз заранее известно, что конфигурация электродов в виде конических сегментов круговых конусов и клиновидных сегментов плоскостей с клиновидными щелями порождает коническое электростатическое поле, однородное по Эйлеру с нулевой степенью однородности $[34,35]$. Однако численный расчет двумерной краевой задачи с последующим пересчетом полученной двумерной гармонической функции в трехмерное электрическое поле выполняется быстрее и обеспечивает лучшую точность.

\section{8. Численные результаты}

В качестве иллюстрации тезисов, высказанных в предыдущих двух разделах, рассмотрим поворотную систему, показанную на рис. 4. Она состоит из вертикальной заземленной плоскости с клиновидной щелью, тангенс угла раствора которой равен 0.09 , и отражающего электрода в виде половины пирамиды с тангенсами угла раствора в плоскостях $O Y Z$ и $O X Z$ равными 0.6 и 1.35 соответственно, и главной осью, совпадающей с координатной осью $O Z$ (рис. 4,a). Эквипотенциальные линии получившегося электрического поля в сечении $z=5$ показаны на рис. 4, $b$. Данная система является огрубленным вариантом электростатических зеркал, показанных на рис. 3. Кроме того, здесь входной (он же и выходной) электрод снабжен бессеточной клиновидной диафрагмой, сохраняющей для краевого электрического поля однородность по Эйлеру нулевой степени. Оптические свойства поворотной системы исследуются численно с помощью программы SIMION 8.1 [36], где для повышения точности расчета электрического поля (весьма существенной в данном случае) используются дробные шаги конечно-разностной сетки.

На рис. 5, а показаны параллельные монохроматические траектории, двигающиеся в плоскости симметрии $O X Z$, которые входят в поворотную систему под углом $82.5^{\circ}$ с начальной безразмерной кинетической энергией $K_{0} / U_{0}=1.095$ (при фиксированных оптических свойствах системы кинетическая энергия нормируется на потенциал отражающего электрода). Несмотря на огрубленную форму электродов и наличие бессеточной диафрагмы и краевого электрического поля, параллельность входного пучка сохраняется на выходе. При численных расчетах точность, с которой для смещенных траекторий воспроизводится выходной угол, была порядка $0.01^{\circ}$.

На рис. 5, $b$ показаны спроектированные на плоскость $O Y Z$ параллельные траектории с тем же самым углом влета и той же самой кинетической энергией, но смещенные вертикально. При этом параллельный 
в вертикальной плоскости пучок на входе системы в телескопическом режиме преобразуется в параллельный пучок на выходе. Наблюдаемый эффект телескопической фокусировки подтверждает устойчивость параллельного пучка при малых отклонениях от плоскости симметрии.

\section{Заключение}

Аналитические электрические потенциалы, однородные по Эйлеру с нулевой степенью однородности и обеспечивающие устойчивость движения заряженных частиц в окрестности плоскости симметрии, которые можно получить с помощью формулы Донкина, являются удобным средством для синтеза электростатических поворотных систем, обладающих свойством преобразовывать входные параллельные монохроматические пучки заряженных частиц (электронов, ионов) в выходные параллельные монохроматические пучки. Такие потенциалы позволяют быстро и эффективно исследовать оптические свойства систем с нетривиальными электродными конфигурациями и отбирать из них те электродные конфигурации, которые обладают перспективными характеристиками с точки зрения той или иной практической задачи. Представляется маловероятным, что подобные системы могут быть найдены с помощью компьютерной оптимизации произвольно взятой начальной конфигурации, если только исследователь не обладает априорной информацией, в каком именно классе систем надо искать оптимальное решение.

Недостатком такой методики является то, что на выходе получаются криволинейные электроды сложной формы, как правило, которые плохо подходят с точки зрения их практического изготовления. Представляется, однако, что замена криволинейных электродов на огрубленные профили в виде клиновидных сегментов плоскостей и конических сегментов круговых конусов, которые описываются в разд. 7 (и иллюстрируются численным примером в разд. 8), будет приводить к электродным конфигурациям с характеристиками, достаточно близкими к характеристикам аналитически рассчитанной системы.

Следует подчеркнуть, что электрическое поле, создаваемое огрубленными электродами с конической геометрией, по-прежнему будет однородным по Эйлеру с нулевой степенью однородности. Значит, хотя такие оптические характеристики, как угол поворота, коэффициент телескопического увеличения, угловая дисперсия по энергии и другие аберрационные коэффициенты огрубленной системы, а также соответствующие огрубленной системе оптимальные напряжения, углы влета пучка и прочие параметры, и будут отличаться от соответствующих параметров аналитической системы, базовое свойство преобразовывать параллельные монохроматические входные пучки в строго параллельные монохроматические выходные пучки останется неизменным.
Основные результаты работы.

1. Поставлена и решена задача адаптации формулы Донкина к синтезу электрических полей, однородных по Эйлеру с нулевой степенью однородности, которые обеспечивают глобальную устойчивость движения заряженных частиц при малых отклонениях от оси симметрии.

2. Обоснована работоспособность принципа подобия траекторий для кусочно- однородных электрических полей, составленных из независимых фрагментов конического типа с одинаковой степенью однородности и непрерывным поведением электрического потенциала на границах между фрагментами (где одним из фрагментов может быть бесполевое дрейфовое пространство, если объект исследования либо точка детектирования вынесены за пределы поля).

3. Приведены примеры монотонно возрастающих аналитических электрических потенциалов с нулевой степенью однородности по Эйлеру, которые могут быть использованы для организации электростатических зеркал, изменяющих направление движения параллельного пучка заряженных частиц. Потенциалы получены с помощью формулы Донкина и обеспечивают глобальную устойчивость для движения пучка заряженных частиц вблизи плоскости симметрии электрического поля.

4. Показано, как с помощью формулы Донкина можно синтезировать электростатические зеркала, использующие однородные электрические поля с нулевой степенью однородности, для которых входным (и одновременно выходным) электродом является заземленная вертикальная плоскость.

5. Показано, что для электростатических зеркал, использующие однородные электрические поля с нулевой степенью однородности, может быть организован бессеточный вход и выход, обеспечивающий для краевых электрических полей однородность по Эйлеру. Такие бессеточные электродные конфигурации в полном объеме сохраняют базовое свойство преобразования входных параллельных моноэнергетических пучков заряженных частиц в выходные строго параллельные моноэнергетические пучки.

6. Показана возможность создания электростатических зеркал, характеризуемых электрическими полями, однородными по Эйлеру с нулевой степенью однородности, с помощью электродов, представляющих собой фрагменты круговых конусов с вершинами в начале координат и плоскостей, проходящих через начало координат.

7. Работоспособность предложенного подхода проиллюстрирована на примере численно рассчитанной поворотной системы, состоящей из вертикальной заземленной плоскости с клиновидной щелью и отражающего электрода, имеющего форму половины пирамиды с прямоугольным основанием и составленного из клиновидных плоских элементов. 


\section{Благодарности}

Авторы благодарны создателям, сотрудникам и спонсорам сайта rspl.royalsocietypublishing.org (Proceedings of the Royal Society of London), в результате самоотверженной работы которых имеется возможность свободно знакомиться с уникальными и раритетными ссылками в частности, с публикациями $[8,9]$.

При выполнении численных расчетов использовалась программа SIMION 8.1 [36]. Для проведения и проверки аналитических выкладок, а также для изготовления рисунков использовалась программа Wolfram Mathematica версии 11 [37]. При редактировании рисунков использовалась свободно распространяемая программа Paint.NET версии 4 [38].

Работа завершена с использованием идей и рукописных черновиков проф. Ю.К. Голикова, но без его прямого участия. Поэтому за все имеющиеся в ней неточности и ошибки несут ответственность исключительно его соавторы.

Авторы благодарны рецензенту за тщательную, вдумчивую и с большим уважением к трудам покинувшего нас Ю.К. Голикова выполненную работу по рецензированию. Авторы также благодарны рецензенту за многочисленные полезные замечания и за фактически состоявшуюся между нами в форме анонимной переписки развернутую научную дискуссию (которая частично вошла в работу), что позволило значительно переработать и улучшить текст.

\section{Финансирование работы}

Работа частично выполнена в рамках НИР 0074-2019-0009, входящей в состав государственного задания № 075-00780-19-00 Министерства науки и высшего образования Российской Федерации.

\section{Конфликт интересов}

Авторы заявляют, что у них нет конфликта интересов.

\section{Список литературы}

[1] Габдуллин П.Г., Голиков Ю.К., Краснова Н.К., Давыдов С.Н. // ЖТФ. 2000. Т. 70. Вып. 2. С. 91-94.

[2] Габдуллин П.Г., Голиков Ю.К., Краснова Н.К., Давыдов С.Н. // ЖТФ. 2000. Т. 70. Вып. 3. С. 44-47.

[3] Фихтенгольи, Г.М. Курс дифференциального и интегрального исчисления. Т. 1. М.: Физматлит, 2001. 616 с.

[4] Смирнов В.И. Курс высшей математики. Т. 1. СПб.: БХВПетербург, 2008. 616 с.

[5] Бердников А.С., Аверин И.А., Краснова Н.К., Соловьёв K.B. // Вестник Актюбинского регионального гос. унта им. К. Жубанова. Физико-математические науки. 2016. № 2 (54), июнь. С. 147-164.

[6] Бердников А.С., Аверин И.А., Краснова Н.К., Соловьёв К.В. // Успехи прикладной физики. 2017. Т. 5. № 1. C. $10-27$.
[7] Бердников А.С., Аверин И.А., Краснова Н.К., Соловьёв K.B. // Вестник Актюбинского регионального гос. унта им. К. Жубанова. Физико-математические науки. 2016. № 2 (54), июнь. С. 17-32.

[8] Donkin W.F. On the Equation of Laplace's Functions \&c. // Philosophical Transactions of the Royal Society of London. 1857. Vol. 147. P. 43-57.

[9] Donkin W.F. On the Equation of Laplace's Functions \&c. // Proceedings of the Royal Society of London. 1856-1857. Vol. 8. P. 307-310.

[10] Гобсон E.B. Теория сферических и эллипсоидальных функций. М.: ИИЛ, 1952. 476 с.

[11] Уиттекер Э.Т., Ватсон Джс. Курс современного анализа. Ч. 2: Трансцендентные функции. М.: ГИФМЛ, 1963. 516 с.

[12] Голиков Ю.К. // Вестник Актюбинского регионального гос. ун-та им. К. Жубанова. Физико-математические науки. 2016. № 2(44), июнь. С. 165-181.

[13] Голиков Ю.К. // Вестник Актюбинского регионального гос. ун-та им. К. Жубанова. Физико-математические науки. 2016. № 2 (54), июнь. С. 59-62.

[14] Голиков Ю.К., Уткин К.Г., Чепарухин В.В. Расчет элементов электростатических электронно-оптических систем: учебное пособие. Л.: Изд-во Ленинградского политех. инта, 1984. $79 \mathrm{c.}$

[15] Галль Л.Н., Голиков Ю.К. // Научное приборостроение. 1987. T. 24. № 1. C. 11-17.

[16] Голиков Ю.К., Краснова Н.К. // Прикладная физика. 2007. № 2. C. 5-11.

[17] Аверин И.А., Бердников А.С., Галль Н.Р. // Письма в ЖТФ. 2017. Т. 43. Вып. 3. С. 39-43.

[18] Голиков Ю.К., Краснова Н.К. Теория синтеза электростатических энергоанализаторов. СПб.: Изд-во Политех. унта, 2010. $409 \mathrm{c}$.

[19] Краснова Н.К. Теория и синтез диспергирующих и фокусирующих электронно-оптических сред. Канд. дис. 01.04.04. СПб., 2013. 259 с.

[20] Голиков Ю.К., Бердников А.С., Антонов А.С., Краснова Н.К., Соловьёв К.В. // ЖТФ. 2018. Т. 88. Вып. 11. C. $1711-1719$.

[21] Голиков Ю.К., Холин Н.А., Шорина Т.А. // Научное приборостроение. 2009. Т. 19. № 2. С. 13-34.

[22] Григорьев Д.В. Электростатические конфигурации высокого энергетического разрешения. Канд. дис. СПб., 2000.

[23] Siegbahn K., Kholine N., Golikov G. // Nucl. Instrument. Methods Phys. Res. A. 1997. Vol. 384. N 1-2. P. 563-574.

[24] Бердников А.С., Краснова Н.К. // Научное приборостроение. 2015. Т. 25. № 2. С. 69-90.

[25] Бердников А.С. // Научное приборостроение. 2015. Т. 25. № 1. C. 48-64.

[26] Краснова Н.К. // ЖТФ. 2011. Т. 81. Вып. 6. С. 97-103.

[27] Голиков Ю.К., Краснова Н.К. // ЖТФ. 2011. Т. 81. Вып. 2. C. 9-15.

[28] Аверин И.А., Бердников А.С. // Успехи прикладной физики. 2016. Т. 4. № 1. С. 5-8.

[29] Голиков Ю.К., Бердников А.С., Антонов А.С., Краснова Н.К., Соловьёв К.В. // ЖТФ. 2018. Т. 88. Вып. 4. С. 609 613.

[30] Коппенфельс В., Штальман Ф. Практика конформных отображений. М.: ИИЛ, 1963. 407 с.

[31] Лаврентьев М.А., Шабат Б.В. Методы теории функций комплексного переменного. М.: Наука, 1965. 716 с. 
[32] Лаврик В.И., Савенков В.Н. Справочник по конформным отображениям. Киев: Наук. думка, 1970. 252 с.

[33] Фильчаков П.Ф. Приближённые методы конформных отображений. Киев: Наук. думка, 1964. 532 с.

[34] Кельман В.М., Карецкая С.П., Федулина Л.В., Якушев Е.M. Электронно-оптические элементы призменных спектрометров заряженных частиц. Алма-Ата: Наука, 1979.

[35] Кельман В.М., Родникова И.В., Секунова Л.М. Статические масс-спектрометры. Алма-Ата: Наука, 1985. 264 с.

[36] SIMION: Ion and Electron Optics Simulator // URL: $\mathrm{http}: / /$ simion.com.

[37] Wolfram Mathematica: the system for modern technical computing // URL: http://wolfram.com/mathematica/.

[38] Paint.NET: Free Software for Digital Photo Editing // URL: http://www.getpaint.net. 